\title{
Une série de disques sur le dhrupad de l'Inde du Nord
}

Dagar Brothers/R. Fahimuddin Dagar, dhrupad vocal/Dagar Duo/F Wasifuddin Dagar, dhrupad vocal

\section{Jane Harvey}

Traducteur : Ramèche Goharian

\section{OpenEdition}

\section{Journals}

Édition électronique

URL : http://journals.openedition.org/ethnomusicologie/764

ISSN : 2235-7688

Éditeur

ADEM - Ateliers d'ethnomusicologie

Édition imprimée

Date de publication : 1 janvier 2001

Pagination : 256-259

ISBN : 2-8257-0723-6

ISSN : 1662-372X

Référence électronique

Jane Harvey, « Une série de disques sur le dhrupad de l'Inde du Nord », Cahiers d'ethnomusicologie [En ligne], 13 | 2001, mis en ligne le 09 janvier 2012, consulté le 20 avril 2019. URL : http://

journals.openedition.org/ethnomusicologie/764

Ce document a été généré automatiquement le 20 avril 2019.

Tous droits réservés 


\section{Une série de disques sur le dhrupad de l'Inde du Nord}

Dagar Brothers/R. Fahimuddin Dagar, dhrupad vocal/Dagar Duo/F Wasifuddin Dagar, dhrupad vocal

Jane Harvey

Traduction : Ramèche Goharian

\section{RÉFÉRENCE}

Dagar Brothers (N. Zahiruddin Dagar \& N. Faiyazuddin Dagar), dhrupad vocal - Raga Miyan ki Todi; Mohan Shyam Sharma (pakhavaj). Enregistré en 1988; durée: 75’45”. 1 CD Jecklin-Disco JD 628-2.

R. Fahimuddin Dagar, dhrupad vocal - Raga Kedar; Lakshminarayan Pawar (pakhavaj). Enregistré en 1989; durée: 79'44". 1 CD Jecklin-Disco JD 635-2.

Dagar Duo (N. Zahiruddin Dagar \& F. Wasifuddin Dagar), dhrupad vocal - Raga Behag; Mohan Shyam Sharma (pakhavaj). Enregistré en 1989; durée: 75'15". 1 CD Jecklin-Disco JD 642-2.

F Wasifuddin Dagar, dhrupad vocal - The Art of Dagarvani Dhrupad: 9 ragas of Night and Day; Praveen Arya (pakhavaj). Enregistré en 1999. Durée totale: 6h17'22”. 5 CD Jecklin Edition JD 721-2.

1 En publiant cette collection de huit $\mathrm{CD}$, Jecklin rend un vibrant hommage à l'art de la grande famille de musiciens Dagar. Jusqu'à la mort de Faiyazuddin en 1989, sept piliers de la vieille génération des Dagar se présentèrent activement en concert en Inde et à l'étranger, dont trois sont enregistrés ici: R. Fahimuddin Dagar (né en 1927), N. Zahiruddin Dagar (né en 1932) et N. Faiyazuddin Dagar (né en 1934). Le quatrième chanteur de cette série de disques, F. Wasifuddin Dagar (né en 1969) est le fils de Faiyazuddin. Après la mort de son père, il quitta sa place derrière la tanpura pour venir 
rejoindre son oncle Zahiruddin sur le devant de la scène et former ainsi le Duo Dagar (CD 3).

2 Malheureusement, Z. Mohiuddin Dagar (rudra vina) décéda en 1990, suivi de son cousin Zahiruddin (oncle de Wasifuddin) en 1994, ne laissant après eux que quatre aînés de la famille Dagar. Hormis Fahimuddin dont il sera question plus bas, on trouve les chanteurs N. Aminuddin Dagar (né en 1923), Z. Fariduddin Dagar (né en 1933) et H. Sayeeduddin Dagar (né en 1939). Aux survivants actifs de la famille se joignent maintenant le fils de Mohiuddin, Z. Bahauddin Dagar (rudra vina), Wasifuddin Dagar (déjà mentionné) et plusieurs disciples de la famille Dagar devenus depuis des artistes reconnus comme les frères Gundecha et Uday Bhawalkar (chanteurs) qui contribuent à perpétuer la tradition.

3 La famille Dagar et ses disciples interprètent un genre ancien de la musique classique de l'Inde du Nord appelé dhrupad. Ils ne sont certainement pas les seuls dhrupadiya (interprètes de dhrupad) de l'Inde, mais les plus connus par les Occidentaux grâce à leurs nombreuses tournées internationales depuis les années soixante jusqu'à nos jours. Les autres dhrupadiya connus en Occident sont notamment Asad Ali Khan (rudra vina) et la famille Mallik (chanteurs). Depuis quelques décennies, la tradition du dhrupad connaît une certaine renaissance. On la croyait en danger d'extinction étant donné que ses interprètes ne sont qu'une poignée comparés à la vaste majorité des musiciens jouant dans un genre plus moderne appelé khayal (pour chanteurs) et un équivalent instrumental du khayal. Toutefois, seules les vingt-cinq prochaines années nous diront s'il y a aujourd'hui suffisamment de jeunes qui sont intéressés et assez dévoués pour se former dans la tradition dhrupad et assurer par là sa survie.

4 Mais revenons à la musique de ces enregistrements. Le premier volume de la liste cidessus, avec le raga du matin Miyan Ki Todi chanté par Zahiruddin et Faiyazuddin Dagar, est un vrai régal. La pureté de l'intonation, ce trait important du style de la famille Dagar, est bien maintenue, associée à cette manière très raffinée et d'une grande beauté de l'interprétation, combinant et ornementant les notes dans le mouvement alap. Dans la section alap d'une interprétation de dhrupad (qui dure 61'54' dans cet enregistrement), le raga est développé lentement et graduellement, sans accompagnement de percussion. Par exemple, la cinquième note, $P a$, qui joue un rôle réduit mais néanmoins significatif dans le Miyan Ki Todi, n'est introduite ici dans l'octave moyenne qu'à la dix-huitième minute, et la tonique supérieure, $S a$, n'apparaît qu'après 29 minutes. Par contraste, la composition de dhrupad avec accompagnement de pakhavaj ne dure que 13'52"; elle est en Chautal, un cycle rythmique de douze temps.

5 L' impopularité relative du dhrupad sur la scène de la musique classique de l'Inde du Nord - où le khayal est préféré comme forme vocale classique - dépend peut-être de goûts musicaux différents en ce qui concerne la manière dont la progression mélodique du raga est exprimée. La révélation lente qu'implique le style dhrupad ne semble pas satisfaire bon nombre de spécialistes de la musique classique. Les autres reconnaissent la valeur du dhrupad, le respectent comme une forme plus ancienne, mais accordent leur préférence aux interprètes du khayal. L'interprétation en khayal du raga comprend d'habitude une courte introduction mélodique (auchar) sans percussion, qui dure de trente secondes à trois minutes et n'expose que quelques-unes des phrases caractéristiques du raga. Ensuite le raga est exploré de façon plus complète dans le barhat (ou vistar), une section initiale de l'interprétation avec accompagnement de tabla. Le style dhrupad de l'exposition du raga conserve dans une certaine mesure sa popularité dans la musique instrumentale, où la 
moitié du temps d'interprétation d'un raga peut être consacrée à un alap sans accompagnement rythmique.

6 Le deuxième CD, Raga Kedar par Fahimuddin Dagar, est celui que je préfère dans cette série. Le raga se déploie lentement dans l'exposition de l'alap claire et détendue de Fahimuddin, alors que les phrases caractéristiques de Kedar sont présentes dès le début, d'abord dans l'octave inférieure, puis dans le timbre de voix différent de l'octave médiane. Le raga Kedar est comme une histoire dont l'intrigue est déjà connue (par les connaisseurs dans le public) avec des phrases nettement définies et des possibilités d'improvisation, mais ses sinuosités particulières ne se dévoilent qu'au moment où elles sont chantées. Sur ce disque, l'alap dure environ cinquante-deux minutes; il est suivi de deux compositions (avec accompagnement au pakhavaj) d'une durée de seize et douze minutes, respectivement en Chautal (12 temps) et Dhamar (14 temps). Personnellement j'aime cet équilibre où la partie avec accompagnement rythmique couvre plus du tiers de l'ensemble de l'interprétation, et aussi le fait qu'il y ait deux compositions. Toute composition traditionnelle dans un raga dévoile un aspect différent de celui-ci. Il est possible que les raga aient tiré leur origine des collections de compositions dont les mélodies similaires ont été ensuite regroupées, donnant ainsi naissance au raga.

7 Sur le troisième $\mathrm{CD}$, le raga Behag (ou Bihag) est chanté par Zahiruddin et Wasifuddin Dagar, l'oncle et le neveu. Dans l' alap, le jeu est plutôt contenu quoique délicat et raffiné. En ce qui concerne l'interprétation du raga, le tivra Ma (quarte augmentée) est l'objet d'une attention que je n'ai jamais entendue dans l'exposition du Behag (il y a aussi une quarte naturelle dans ce raga). A l'extrême opposé, certains musiciens préfèrent réduire l'usage du tivra $M a$ à une très légère suggestion de temps à autre. Ce faisant ils traitent cette note comme la jolie fleur de belladone qu'il faut regarder sans la toucher! Dans ce CD, 59'30" sont consacrés à l'alap, suivies d'une composition Chautal qui est un duo vocal plein d'entrain avec un accompagnement au pakhavaj très animé par Mohan Shyam Sharma. Le tambour pakhavaj à deux membranes est un instrument merveilleux; c'est parfois dommage que l'on doive attendre si longtemps dans la première partie d'un récital de dhrupad avant qu'il n'intervienne.

8 L'art du Dhrupad Dagarvani, neuf raga du jour et de la nuit chantés par Wasifuddin Dagar (dans un coffret de cinq CD), commence par le raga de l'aube Lalit. Il se déroule du matin jusqu'à la nuit avec les raga Hindol, Shuddh Saarang, Pooria, Eman, Chandrakauns, Darbari Kanada et Adana jusqu'au raga d'après-minuit Sohni. Le temps d'écoute s'étend sur 6h17'22" et peut donc occuper une grande partie de la journée. Cependant, il faut vraiment prendre son temps pour écouter ce $\mathrm{CD}$ et ne pas l'entendre uniquement comme musique de fond. Il est préférable de prendre un ou deux raga par jour (sans jeu de mots d'ordre médical)!

9 Pour moi, en tant qu'auditrice, il y a trois catégories de musique classique:

1. Une musique qui enchante, apprend et ravit;

2. Une musique correctement interprétée mais ennuyeuse (ce qui peut aussi être subjectif);

3. Une musique qui est mal interprétée et mal jouée ou simplement de la mauvaise musique (même si elle est jouée par de très bons musiciens). Face à cette catégorie, je tâche de m'en aller avant la fin du concert.

10 Je dois avouer que tous les récitals Dagar que j'ai entendus appartiennent sans équivoque à la première catégorie. Les préférences que j'exprime dénotent uniquement les différents degrés de plaisir ou de concentration méditative auxquels j'ai accédé. 
Confrontée à la splendeur des enregistrements de neuf merveilleux raga chantés par Wasifuddin Dagar dans la paix du temple de la famille Holkar à Maheshwar, en Inde, je sens que j'aime certains d'entre eux encore plus que les autres, à savoir Sohni, Pooria et Chandrakaus dans lesquels les qualités musicales de Wasifuddin me paraissent s'exprimer le mieux. J'aime Sohni pour la douceur de son timbre, Chandrakaus pour sa complexité inattendue et Pooria pour la plénitude de la voix et l'intensité poignante exprimée dans le raga. Il y a aussi cette composition rapide et passionnante dans Adana, dont le texte est dédié à Shiva et qu'il ne faut absolument pas manquer.

11 Les livrets des CD montrent des photos des musiciens en action et donnent une intéressante information de fond malgré quelques incohérences mineures. Par exemple, les dates de naissance de Zahiruddin et de Faiyazuddin sont indiquées comme étant 1933 et 1944 dans le livret du Miyan Ki Todi mais (je crois correctement) comme 1932 et 1934 dans l'Art du Dhrupad Dagarvani (les cinq CD du volume de Wasifuddin). Ce dernier présente un arbre généalogique très utile de la famille Dagar ainsi que plusieurs articles érudits et instructifs. On trouve un bon texte sur le dhrupad par Peter Pannke dans le volume du Duo Dagar (Raga Behag). Les livrets des CD numéros 1 à 3 de la liste initiale sont bilingues anglais-allemand et celui des cinq CD n'est qu'en anglais. Tous les livrets donnent une brève description du raga sous forme de mouvements ascendant et descendant en notation occidentale et indienne et ils contiennent également tous les textes et les traductions des compositions. Les notes consacrées aux raga Kedar et Behag indiquent aussi les battements de tambour des cycles rythmiques utilisés. 